\title{
¿LA PSICOLOGÍA HUMANISTA COMO PSEUDOCIENCIA?
}

\section{THE HUMANIST PSYCHOLOGY AS A PSEUDOSCIENCE?}

\author{
Ana Gimeno-Bayón Cobos \\ Ramón Rosal Cortés
}

Instituto Erich Fromm de Psicoterapia Integradora Humanista. Barcelona, España

Cómo referenciar este artículo/How to reference this article:

Gimeno-Bayón Cobos, A. y Rosal Cortés, R. (2019). ¿La Psicología Humanista como Pseudociencia?. Revista de Psicoterapia, 30(112), 165-188. https://doi.org/10.33898/rdp.v30i112.285

\begin{abstract}
Resumen
A la vista de la aparición del término "pseudociencias" en los medios de comunicación, en relación con algunos métodos y modelos de intervención en psicoterapia, el presente artículo pretende dar cuenta de algunos criterios a la hora de entender la calidad cientifica en este ámbito. Igualmente quiere abogar por la coexistencia de un pluralismo de modelos, cuya diversidadlejos de una rigidez uniformadora y dogmatizante en favor de uno solo de ellos-comporta una riqueza de paradigmas psicológicos y psicoterapéuticos. La posibilidad de convivencia de éstos, en un momento dado, favorece un diálogo que hace progresar el conocimiento, sin restar credibilidad en cuando a la calidad cientifica de los mismos. En el artículo también se aborda la diferencia existente entre lo que se entiende por evidencia cientifica en las ciencias naturales como, por ejemplo, en Biología o Medicina- y en las ciencias humanas (o sociales) como es el caso de la Psicología y la psicoterapia. En cuanto a esta última, se citan algunos de los resultados y conclusiones a los que se ha llegado, y las propuestas acerca de nuevos paradigmas de investigación que se apuntan en este campo.
\end{abstract}

Palabras clave: Psicoterapia, paradigma cientifico, investigación, evidencia, pseudociencias, psicoloCorrespondencia sobre este artículo:

E-mail: anagb.ifromm@gmail.com y ramonrosalc@gmail.com

Dirección postal: c. Madrazo 113, enlo. 2ª 08021 Barcelona. España

(C) 2019 Revista de Psicoterapia

\begin{abstract}
In view of the appearance of the term "pseudosciences" in the media, in relationship with some methods and intervention models in Psychotherapy, the article aims to explain various criteria to understand the scientific quality of this field. At the same time it wants to defend the pluralism of models, whose diversity -too far from an uninforming and dogmatizing rigidity in favor of one of them-implies wealth in psychologic and psychotherapeutic paradigms. The possibility that these could coexist in a certain point in time, favors a dialog that makes knowledge progress without removing any credibility from its scientific quality. In the article it is also talked about the existing difference between scientific evidence in natural sciences - as for example Biologyor Medicine-and in human (or social) sciences as is the case of Psychology and Psychotherapy. In connection with psychotherapy, it quotes some of the results that have been reached and the proposals of new paradigms of investigation.
\end{abstract}

Keywords: Psychotherapy, scientific paradigm, research, evidence, pseudosciences, humanistic psychology.

Fecha de recepción: 08/01/2019. Fecha de aceptación v1: 15/01/2019. Fecha de aceptación v2: 30/01/2019. 
Ante algunos acontecimientos recientes, que lógicamente han alarmado a la comunidad científica, y en los que se cuestiona la validez de la Psicología Humanista y las psicoterapias encuadradas en este paradigma, y que corren el peligro de ser tildadas de "pseudociencias" (Plan para la protección de la salud frente a las pseudoterapias, presentado por parte de los Ministerios de Sanidad, Consumo y Bienestar Social y el Ministerio de Ciencia, Innovación y Universidades , presentado el pasado 14 de noviembre) se hace necesario manifestar nuestras reflexiones en torno al tema. Dado que la justificación que alega ese plan es que algunos modelos o metodologías de la Psicología o Psicoterapias Humanistas no reúnen los requisitos científicos de evidencia empírica, este será el foco que centrará la atención de este escrito.

Al querer proteger a la ciencia de sus posibles falsificaciones, habría que evitar dar la impresión de que se presupone que solo hay un modelo válido de ciencia: el modelo vigente y casi exclusivo en el mundo académico español. Es decir, un modelo de ciencia psicológica y psicoterapéutica que constituyó una aportación valiosa de la corriente conductista que tuvo casi el monopolio en las Facultades de Psicología en España más o menos hasta los años sesenta del pasado siglo y que posteriormente ha sido sucedido por el modelo cognitivo-conductual. Este último ha logrado captar y respetar mucho mejor que el conductista la complejidad del psiquismo humano: no ha pretendido reducir los procesos psíquicos a procesos fisiológicos. La principal y casi única herencia que ha recogido del conductismo es su metodología científica, aunque renunciando a la utilización predominante de ratas u otros animales en sus trabajos de investigación. En cambio, las llamadas "psicoterapias de tercera generación", procedentes del modelo cognitivo-conductual no han tenido ningún empacho en tomar bastantes de sus técnicas de intervención de los modelos de psicoterapias humanistas (eso sí, sin nombrarlos). Así ocurre, por ejemplo, con la utilización de determinadas técnicas de actividad imaginaria, o de Mindfulness, de aceptación de la intención positiva del síntoma, etc.

Como es sabido, fueron surgiendo, a lo largo del siglo XX, una larga serie de nuevos modelos de psicoterapia, entre los cuales se encuentran los que pueden considerarse vinculados a la corriente humanista, también denominada existencialhumanista, o experiencial-humanista. Estas últimas denominaciones son debidas a la importancia concedida en algunos modelos terapéuticos humanistas a los problemas existenciales, y a la relevancia de los trabajos experienciales durante las sesiones terapéuticas.

Como elementos del denominador común dentro del pluralismo de las psicoterapias humanistas podemos destacar:

a) El claro predominio de las concepciones holista y sistémica frente a las atomistas y reduccionistas. No se acepta la explicación de la conducta humana a partir exclusivamente de factores elementales como estímulos, impulsos o cualesquiera otros.

b) Se reconoce la libertad de elección y de creatividad, frente a concepciones 
mecanicistas y deterministas.

c) Se investiga a personas con un alto grado de salud mental o autorrealización, y no solo a las afectadas por psicopatologías.

d) Otro elemento de su denominador común es el rechazo a compartir la vigente sobrevaloración de la importancia de un tipo de metodología cientifica concebida para las ciencias naturales.

Esta metodología había sido concebida para el estudio de los hechos de los que se ocupan las ciencias físicas, químicas o biológicas, y trata de cuantificar los fenómenos que observa y de identificar variables dependientes e independientes, partiendo de la suposición de que todo puede explicarse según un proceso de causalidad lineal. Hay que tener en cuenta que por aquellos años las metodologías científicas utilizadas en Psicología no habían alcanzado, en sus modalidades observacionales y cualitativas, el rigor y la riqueza de matizaciones con las que puede contemplar en la actualidad los procesos psíquicos humanos (véase Anguera 1981a, 1981b, 1986, 1995).

Tal sobrevaloración de las posibilidades de la metodología científica de los años cincuenta para el abordaje de las investigaciones psicológicas, había dado lugar a advertencias de científicos destacados, como el físico y premio Nobel Heisenberg, cuando afirmaba que consideraba una ilusión la creencia en la posibilidad de observar la naturaleza -en especial la humana- como un objeto externo separado del sujeto, y se lamentaba de que en la Psicología se fomentase esta actitud, cuando en esta ciencia, al tener que utilizar el investigador los mismos procesos cuyos mecanismos y estructura trata de descifrar, la simplificación que puede producirse resulta incomparablemente más grave que en la Física (Rosal, 2001, pp. 24s.).

Los investigadores científicos de la Psicología Humanista respetan los requisitos de la metodología convencional heredada del conductismo, pero solo la consideran aceptable para la investigación de algunos aspectos puntuales de las conductas. Comprueban que para otros de los más interesantes, esa metodología resulta inadecuada. Es como ponerse gafas para oír mejor. Por mucho que se logre la graduación perfecta, no se conseguirá mejorar lo que se oye. Como han puesto de relieve algunos investigadores (Charman y Barkham, 2005; Margison et al., 2000), los diferentes paradigmas necesitan métodos de investigación diferentes, que pueden ser complementarios entre sí. Y la Psicología Humanista, necesita para su investigación de unos métodos que sirvan a su específica manera de realizar la psicoterapia. Como señala Cencillo (año):

El modo exclusivo de tratar cientificamente cualquier objeto no puede ser otro que descomponerlo en sus últimos elementos sensorialmente observables, es decir, tratándose del hombre, sus células, tejidos y, a lo sumo, órganos, pero no los campos de influencias sociales y culturales, la comunicación y la afectividad, pues nada de esto es sensorialmente observable ni simple [...] Tolman, en 1932, ya vio que no podía quedar la investigación de la conducta en unidades "moleculares", sino que 
había que referirse a totalidades "molares" (Cencillo, 1977, p. 23).

Naturalmente, cuando el autor dice "científicamente" lo está haciendo desde un punto de vista irónico, burlándose de la cientificidad reduccionista mantenida por la ciencia médica y psiquiátrica. Este autor señala que:

El cientifismo actual, desgraciadamente aplicado a las ciencias humanas (pues las ciencias físicomatemáticas han conquistado una libertad y una amplitud de recursos de verificación y de modelización inconmensurablemente mayor), no parece considerar como válidos, sino, exclusivamente,

- percepción sensorial

- medibilidad espacio-temporal

- modelos lógico-matemáticos clásicos

Y precisamente la percepción sensorial (aunque esté potenciada por instrumentos específicos) acabó por ser, desde los resultados de la Física, lo menos directo, más mediatizado y más cuestionable en su fiabilidad de cuantos canales de comunicación con la realidad dispone el hombre (Cencillo, 1977, p. 14) (Cfr. además, Cencillo, 1978).

Toda ciencia del hombre verdaderamente tal ha de poseer unos recursos propios -y no solamente prestados por otras ciencias ajenas al objeto "hombre" y necesariamente distintos de los de las ciencias unidimensionales de la materia no humana, para investigar, localizar, controlar y modelizar sistemáticamente los niveles y factores específicos de tal objeto (Ibidem, p. 32).

\section{Distintos Paradigmas, Distintas Metodologías}

A la Psicología Humanista no le sirve el modelo médico, que es el que convencionalmente se entiende como válido para investigar fenómenos humanos. Y no le sirve porque la Medicina opera en el campo de las Ciencias Naturales, mientras la Psicología opera en el campo de las Ciencias Humanas, también llamadas Ciencias Sociales. Mientras en el primero se busca estructurar los hechos en forma escrupulosamente rígida y objetiva, en el segundo se busca interpretarlos en forma adecuada; mientras en el primero se procura limitar la subjetividad, en el segundo ya da por sentado que, como trabaja precisamente con la subjetividad, no puede "demostrar objetivamente" nada, sino formular hipótesis verosímiles.

Hay que tener en cuenta también que la dinámica científica opera en forma distinta en ambos campos:

a) En las ciencias naturales hay un paradigma único, que se va enriqueciendo con sucesivas aportaciones, y que si es cuestionado repetitivamente, es sustituido por un nuevo paradigma que integra el anterior.

b) En las ciencias sociales pueden convivir varios paradigmas, todos ellos igual de verosímiles, que pueden ir enriqueciéndose cada uno de ellos con sucesivas aportaciones en el seno de cada paradigma, y del diálogo con los otros paradigmas. A. Caparrós, el que fue catedrático del Historia de la Psicología y también rector de la Universidad de Barcelona, y que se 
refirió siempre con respeto a la Psicología Humanista, sostuvo el carácter pluriparadigmático de la ciencia psicológica (vid. Caparrós, 1978 y 1980). A su aportación se refiere el capítulo titulado El movimiento de la Psicología Humanista y su relación con la Psicología Científica (Rosal, 2001), ampliación de la ponencia presentada en 1981 en el Primer Congreso Nacional de Psicología Humanista, celebrado en Barcelona.

En el ámbito de las ciencias naturales se trabaja con hechos físicos, mientras en el segundo se trabaja con las motivaciones, la conciencia, la subjetividad y la intencionalidad, que no pueden ser susceptibles de observación externa; en el primero se trabaja con experimentos que se pueden repetir con los mismos objetos o sujetos, buscando la posibilidad de generalización. Si tal intervención demuestra ser eficaz-según la evidencia científica- se puede generalizar y aplicar a todas las situaciones similares en la confianza de que será igualmente eficaz.

En el segundo ámbito son irrepetibles, como lo son los sujetos, e incluso los mismos sujetos son irrepetibles en los distintos momentos ya que, como señala William James (1989) como cada experiencia modifica el cerebro, aunque se repita la misma experiencia, esta tiene lugar en un cerebro modificado, por lo que tiene un carácter único. Quizás es por la intuición de su irrepetibilidad en el campo de la investigación psicológica por lo que tan solo un 1\% de las investigaciones en Psicología (desde inicios del pasado siglo) son replicaciones de estudios anteriores, como señala el metaanálisis de Makel, Plucker y Hegarty (2012). Y quizás por eso mismo la "crisis de la replicación" -especialmente desde que se descubrieron los fraudes de Stapel (Shea, 2011)- que afecta muy especialmente al mundo de la Psicología científica, dado el bajo éxito obtenido en las replicaciones, no es algo que obedezca simplemente al llamado "efecto cajón" -por el que las investigaciones que no verifican la hipótesis planteada no se publican, y sí en cambio las que la verifican (con lo que no se da una transparencia del conjunto de la investigación), ni al fraude a que nos tienen acostumbrados las investigaciones (en el campo médico-farmacéutico), sino a que igual es algo intrínseco al campo de la Psicología y hay que contar con ello. Baste señalar que Camerer et al. (2018) replicaron 21 experimentos de Psicología publicados entre 2010 y 2015 en las más prestigiosas revistas, Nature y Science, de los que solo 13 tuvieron éxito. A no ser que el sesgo de confirmación y el contexto de la relación esté de por medio y explique por qué, en la investigación dirigida por Nosek (Varios, 2015), de 100 estudios en Psicología replicados solo se confirmó un $36 \%$ con resultados significativos, aunque, si en la replicación se encontraba algún miembro del equipo original, ese porcentaje aumentaba.

A este tipo de dificultades propias del ámbito de las ciencias humanas, en el que se encuadra la Psicología, se refería Hoffman para la terapia familiar:

Surge entonces esta pregunta: ¿Hay una hipótesis verdadera? Obviamente, algunas son más "verdaderas" que otras [...] El grupo de Milán se enfrenta a este problema citando el Oxford English Dictionary, que define una hipótesis como 
"suposición hecha como base para razonar, sin referencia a su verdad, como punto de partida para una investigación". [...]

$\mathrm{Si}$ es "viable" en el sentido de una suposición sobre la cual basar un experimento, esto es algo que sólo en retrospectiva puede juzgarse, y aún entonces, retrospectivamente. Para el momento en que una hipótesis parezca justificada por el curso de los hechos, la familia presentará una configuración distinta, lo que significa que la hipótesis original debe ser revisada, o aún totalmente suprimida. (Hoffman, 1987, p. 274).

Es más: en el ámbito de la psicoterapia, en concreto, ámbito complejo e influido por la relación interpersonal, el contexto de la investigación cuenta. Y si es una hora tardía, o el investigador tiene cara de cansado, es joven o viejo, se hace la investigación en lunes o viernes (por no hablar de un día de fiesta) etc. son variables que raramente se pueden controlar y que pueden sesgar el experimento. Pero ya de por sí, como dentro de la deontología de la investigación se incluye el dar cuenta al sujeto de que está participando en una investigación, este hecho puede ser muy determinante del comportamiento y las respuestas del mismo, incluso de una manera no consciente. Es por ello que cualquier intento de investigación intencional desde cualquier metodología convencional en psicoterapia se encuentra en una encrucijada: o falta a la ética (escondiendo su finalidad al sujeto), o falta a la naturalidad del proceso psicoterapéutico y por lo tanto trabaja con una situación falsa. Tan solo a posteriori, y con el consentimiento del sujeto, es factible revisar las observaciones realizadas con menores posibilidades de sesgo en su verificación (del todo, nunca, y de ahí la necesaria complementariedad de métodos psicológicos científicos).

\section{¿Qué Significa "Evidencia Empírica"?}

Para comprender otra gran diferencia de lo que significa "evidencia empírica" en las ciencias naturales y en las ciencias sociales, se va a relatar a continuación un ejemplo absurdo.

Imaginemos un experimento que intenta corroborar con evidencia empírica que las piedras caen hacia abajo debido a la ley de la gravedad. Hay una evidencia experiencial, pero ¿alguien se tomó la molestia de comprobar en un laboratorio que las piedras caen? Newton lo explicó, analizó, encontró la fórmula, pero... ¿comprobó esto mediante un experimento de laboratorio que pudiera acreditar su "evidencia científica"? ¿O más bien buscó entender lo que era una evidencia experiencial?

Se puede suponer que, para comprobar que los cuerpos sólidos caen hacia abajo, se aplica el experimento a 35 piedras, de igual composición, forma y tamaño, en contraste con otro grupo igual de piedras idénticas, a las que se deja en el suelo. ¿Qué pasa si al meter a las piedras en el laboratorio cinco de ellas se evaporan? Nos quedan 30 piedras, que constituirán el 100\% de los objetos con los que experimentar. ¿Y si además otras cinco, también al entrar en el laboratorio, cambian su forma de una manera inexplicable? Ese aspecto no se va a tener en cuenta pues lo que se 
mide no es eso. Imaginemos que el experimento da como resultado que de las 30 piedras objeto del experimento, lanzadas desde una altura de tres metros, 28 caen al suelo, mientras dos no caen y se quedan flotando. Estadísticamente, la evidencia empírica da unos resultados significativos de que de las 30 piedras lanzadas en esas condiciones, 28 caen, por lo que se pueden generalizar sus resultados.

Pues bien: ¿No sería un poco raro osar generalizarlos sin estudiar por qué algunas (aunque pocas) piedras se evaporan en el laboratorio y otras cambian de forma? ¿No sería osado generalizar esos resultados sin investigar qué condiciones hacen que algunas piedras - pocas-no caigan? ¿No sería más interesante, antes que presumir de la evidencia empírica, demostrada científicamente, de que las piedras caen (para lo cual no hacía falta tanto experimento, dada la evidencia experiencial), preguntarse por la estructura subyacente que hace que unas se evaporen o cambien de forma al introducirlas en un laboratorio, y por qué otras no caen, aunque sea según el método científico convencional- en una pequeña proporción estadísticamente poco relevante (dos sobre 30)?

De acuerdo. Ya se había anunciado que ese experimento sería absurdo en el marco de las ciencias naturales. ¿Por qué? Porque las piedras, al meterse en un laboratorio no se evaporan, ni cambian de forma de una manera grosera (aunque Heisenberg, en un nivel sutil, ya demostró al formular el "principio de incertidumbre" que la mirada del investigador, en niveles subatómicos producía cambios, hallazgo por el que recibió el Premio Nobel de Física en 1932). Pero en el marco de las ciencias sociales, tales fenómenos ocurren, y los científicos que en Psicología utilizan el paradigma correspondiente a las ciencias naturales no se preguntan qué estructura subyace para explicar los sujetos que "se evaporan", (desaparecen del experimento porque lo abandonan); ni por qué tres "cambian de forma" (se adaptan para cumplir el deseo implícito intuido del experimentador); ni por qué dos sujetos "no caen", es decir, los resultados dan que no siguen igual norma que los otros. Y eso en el marco de la Psicología y la Psicoterapia es la realidad de todos los experimentos científicos. El intento de generalizar los resultados con evidencia científica, y aplicarlos a toda la población, puede ser inoperante o contraproducente para los 12 sujetos que -de los 35-no cumplieron la hipótesis en sentido riguroso, aunque el registro diga que solo dos no lo cumplieron.

Además, mientras se pueden controlar bien la composición, peso y forma de las piedras, de modo que sean prácticamente idénticas, y la altura desde la que se las lanza, no hay dos sujetos idénticos ni una situación idéntica, de modo que no sería posible generalizar los resultados al resto de la población.

Añadamos a esto que el experimento sirve para las piedras equiparables lanzadas desde tres metros de altura. Haciendo un equivalente psicológico, a las personas que obtienen " $x$ " puntuación tras la aplicación de una batería de pruebas. Pero, dada la precisión y rigor científicos exigibles, no se sabe si el resultado del experimento serviría para las piedras lanzadas desde dos o cuatro metros. No hay evidencia empírica de ello. En psicoterapia, la evidencia empírica que aporta la 
ciencia serviría (en el mejor de los casos) solo para aquellos en que la medida es exactamente la misma que la de la muestra. ¿Y qué pasa con quien no da la medida? ¿Se podría aplicar-de forma poco rigurosa-el tratamiento científicamente evidente para otra medida?

Pero el complejo de inferioridad con el que se desarrolló la Psicología en relación con la Física decimonónica ha hecho que se hayan dedicado muchos esfuerzos de investigadores a intentar demostrar con la metodología de las ciencias naturales obviedades que para el clínico que formula la hipótesis son evidencias constatadas decenas o centenares de veces en su práctica psicoterapéutica. Podrían haberse destinado esa energía y esos recursos al avance real de la teoría clínica de la profesión. Este hecho resulta poco motivador para que el psicoterapeuta investigue con este paradigma, a no ser que sea un profesor universitario de Psicología Humanista, en alguna de las escasas Facultades que la imparten en nuestro país, a los que se les exige publicar.

En psicoterapia no hay dos personas, ni dos momentos, ni dos contextos vitales idénticos. Por ello (además de la propia experiencia y la lectura de casos y experimentos) toca al psicoterapeuta, en el presente del fluir de la sesión terapéutica, utilizar su intuición para decidir qué intervención será eficaz. Esto de la "intuición" parece un concepto poco científico, pero ocurre como con las "meigas": que, aunque no se crea en ellas "haberlas haylas". En realidad se trata de un concepto poco contemplado desde las ciencias naturales y al que los estudios de la Psicología académica ha relegado al cuarto de los trastos (quién sabe si por un sesgo machista ante la connotación que tiene la intuición de "femenina"). Sin embargo, Eric Berne, en sus agudos estudios sobre la intuición (Berne, 2010) pudo concluir cómo, cuando se trata de situaciones complejas (como la simple observación de un rostro humano, ya de por sí muy complejo) la intuición tiene más aciertos que los que derivan de las conclusiones lógicas abstraídas de la observación. La confianza en la aplicación de tales reglas, en lugar de confiar en la intuición, da menos aciertos.

Quizá por haber planteado la investigación sobre la eficacia de la psicoterapia en términos de una metodología que no le era aplicable es por lo que no se encontraron diferencias entre los diferentes tipos de tratamiento (Lambert, Shapiro y Bergin, 1986; Luborsky, Singer y Luborsky, 1975). Este hecho sorprendió y sirvió para cuestionar y hacer nacer nuevos métodos de investigación, a fin de hacerlos más adecuados a los paradigmas dentro de los cuáles tenía lugar la investigación. Pero los diferentes estudios llevaban a la misma conclusión: en psicoterapia -a diferencia de lo que ocurre en las ciencias naturales-no es posible la generalización. Al menos por lo que hace a las escuelas y modelos terapéuticos. Por eso la constatación de que no hay un modelo adecuado para todo tipo de persona y problema dio lugar a un enorme interés por la formulación de Paul (1967) de una matriz -tratamiento x terapeuta x cliente x problema- (es decir qué tratamiento ha de ser aplicado, por qué terapeuta, a qué tipo de cliente y en relación con qué problema). Su aceptación significaba "una revelación clara de que los resultados 
habían ido mostrando que la aplicación incondicional y uniforme de un modelo determinado de tratamiento -independientemente de las variables del terapeuta, del paciente y del problema concreto-, debe ser puesta en cuestión" (Gimeno-Bayón, 2013, p. 222). Como señala la APA (American Psychological Association), asociación que dedica su División 32 a la Psicología Humanista, a propósito de la psicoterapia basada en la evidencia, en su Resoluttion on the Recognition of Psychotherapy Efectiveness de agosto de 2012, "Las comparaciones entre las distintas modalidades de psicoterapia no suelen presentar diferencias significativas $\mathrm{y}$, generalmente suelen estar moderadas por factores contextuales y relacionales".

Por otra parte, la evidencia empírica ¿qué mide exactamente? Convencionalmente, se trata de dilucidar hasta qué punto cumple los requisitos de "las tres "e": eficacia, efectividad y eficiencia, diferenciación necesaria y que parece estar presente en el Plan ministerial citado en el prólogo, pues unas veces habla de una evidencia científica "que avale su eficacia y su seguridad" (p. 3 del Plan para la Protección de la Salud frente a las Pseudoterapias), otras veces habla de que "todavía son muchas las personas que creen que algunos tratamientos son efectivos a pesar de no estar avalados por las evidencias científicas disponibles" (Ibidem) y en otro momento: "los principios inspiradores de la Red son los de seguridad, efectividad, calidad, equidad y eficiencia" (Ibidem, p. 7).

En el marco de la Salud,

Eficiencia: Es el grado en que el sistema de salud efectúa la máxima contribución a las metas sociales definidas dados los recursos disponibles del sistema de salud y de aquellos que no pertenecen al sistema de salud. Implica la relación favorable entre resultados obtenidos y costos de los recursos empleados. Tiene dos dimensiones: la relativa a la asignación de recursos y la referente a la productividad de los servicios.

Eficacia se refiere a los resultados en relación con las metas y cumplimiento de los objetivos organizacionales. Para ser eficaz se deben priorizar las tareas y realizar ordenadamente aquellas que permiten alcanzarlos mejor y más. Es el grado en que un procedimiento o servicio puede lograr el mejor resultado posible. Es la relación objetivos/resultados bajo condiciones ideales

Cuando se crean condiciones de máximo acondicionamiento para alcanzar un fin y este se logra, los recursos puestos en función de este fin fueron eficaces.

Efectividad es la relación objetivos/resultados bajo condiciones reales (Lamb y Hernández, 2008).

Como puede verse, mientras la eficiencia es un criterio de tipo económico (¿compensa la inversión?), la efectividad mide la realidad de los resultados en las condiciones habituales y la eficacia hace referencia a las expectativas respecto a de un determinado tipo de intervención si se dan las condiciones ideales, tanto en su aplicación como en las condiciones experimentales.

Pues bien: en el caso de la búsqueda de evidencia científica en el caso de la salud mental, y en concreto, en el de la psicoterapia, hay que descartar, de entrada, 
la posibilidad de demostrar la eficacia de un determinado modelo o experimento con los criterios de las ciencias naturales, puesto que las condiciones óptimas exigen que no se produzca en el marco de tal experimento, sino en el contexto de un proceso natural, para evitar el "efecto laboratorio" (que las piedras cambien de forma, en el ejemplo antes expuesto). Es por ello por lo que resulta enormemente pertinente la advertencia de Hoffman antes citada. El pensar en equiparar las condiciones óptimas a las condiciones experimentales, en psicoterapia es una burda falacia.

Otra cosa es la posibilidad de estudiar la efectividad y eficiencia de las técnicas de intervención concretas. Intervenciones que dan cuenta, según los estudios de Lambert, Shapiro y Bergin (1986) del 15\% del éxito de la psicoterapia. No hay que olvidar que la intervención concreta se da en el ámbito más amplio de un proceso que sitúa y otorga significación a ese cambio. De por sí, la contribución de una intervención aislada efectiva y eficiente no da cuenta del sentido respecto al conjunto, pero es muy interesante a tener en cuenta en el nuevo paradigma de los cambios intrasesión, sobre todo si se da en el marco de una relación terapéutica efectiva y eficiente, como ha mostrado ser la que propone la Psicología Humanista a partir de las actitudes rogerianas (Rosal, 2013). Las múltiples investigaciones que se han realizado en el ámbito de las psicoterapias experiencial-humanistas mostrando su efectividad (p.e., en el marco de la Psicoterapia Integradora Humanista: Gimeno-Bayón, 1999; Lizeretti, 2009; Oriol, 2014; Rosal, 1997) y su eficiencia (Also, 2017), tienen en cuenta el marco relacional, despegadas del cual seguramente estarían abocadas al fracaso o a la disminución de su éxito.

Se podría decir que, en psicoterapia, la evidencia más clara viene dada por la constatación por parte del sujeto que acudió a ella de haber obtenido los cambios que deseaba. Esa es la mejor prueba científica para el psicoterapeuta, en un proceso único, con una persona única y en un momento único de su vida. Todo lo demás son intentos de justificar, con los requisitos académicos del momento (aunque sean equivocados u obsoletos), la maravilla de asistir a esa creación de una nueva y más sana forma de vivir que ocurrió en el paritorio de la sala de terapia.

\section{La Evolución en el Concepto de "Evidencia Científica"}

Como bien señala Cestari (2016), en Psicología se partió de copiar de la Medicina el método de la Práctica Basada en la Evidencia (PBE) para evitar que el médico no se limitase a los hallazgos de su experiencia individual a la hora de planificar un tratamiento clínico, sino que tuviera el apoyo de pruebas científicas objetivas que le guiase a la hora de tomar decisiones sobre la salud física del paciente.

Ahora bien: esa metodología, procedente del ámbito de las ciencias naturales es inadecuada para resolver los complejos problemas del ámbito de las ciencias sociales, como ya se ha comentado.

Las exigencias de investigación para cumplir con lo que pide este método, resultan absurdas dentro del campo de la Psicología Clínica. Pero, en primer lugar, 
habría que definir a qué se considera "evidencia”, puesto que no hay un solo criterio de la misma. Vera-Villarroel y Mustaca, señalan, por ejemplo:

Para evaluar los tratamientos, los especialistas usan como herramienta fundamental las publicaciones sobre el tema y determinan su validez en función de los criterios metodológicos pre-establecidos. De ese modo, construyen una base de datos que les permite ir discriminando las terapias más eficientes para cada trastorno e ir creando las listas de tratamientos con apoyo empírico (TAEs) que se difunden en libros y en Internet. De esto se deduce que la elaboración de tales listas es dinámica y depende de la cantidad de trabajos publicados. La mayoría de las publicaciones que se usan de referencia para la elaboración de las listas son de revistas norteamericanas, canadienses e inglesas (ej. British Journal of Psychiatry, Journal of Pediatric Psychology, Clinical Psychological Review y Canadian Psychology). No hay referencias a revistas latinoamericanas. Aunque se supone que las TAEs tienen una validez universal porque se basan en los principios básicos de la conducta, hay que reconocer que es un "supuesto" que debería ser contrastado empíricamente en otros países y aumentar así el grado de generalidad de los tratamientos. Algunas diferencias culturales entre los países podrían influenciar cuándo se aplican (Vera-Villarroel y Mustaca, 2006, p.553).

Bien, si el criterio de la evidencia empírica es la cantidad de publicaciones, se puede llegar a la conclusión-que no parece demasiado lógica-que el PBE hace más eficaces las terapias en inglés, o las que predominen en países de esa habla, puesto que hay más trabajos con apoyo empírico en inglés. Aparte de que, como advierte Corey (1995), cómo los diferentes modelos terapéuticos son más o menos apropiados para las distintas culturas.

Aquellos autores destacan también como "un importante intento para evaluar a las terapias psicológicas en EE. y Canadá" la creación de unas guías con la colaboración y auspicio de la División 12 (Psicología Clínica) de la Asociación Psicológica Americana APA" en las que se medía la eficacia de "un tratamiento" a través de tres criterios: a) existencia de al menos dos estudios rigurosos de distintos investigadores que demuestren superioridad al tratamiento farmacológico, al placebo o a otro tratamiento, con diseño experimental intergrupal de un $\mathrm{N}=30$ por grupo como mínimo, o una serie de estudios de caso único ( 9 como mínimo); b) contar con un manual de tratamiento; y c) los sujetos a prueba deben estar claramente identificados en función de un manual, como el DS-IV (Echeburrúa y Corral, 2001; Hamilton y Dobson, 2001) (Vera-Villarroel y Mustaca, 2006, p.552s.).

Para un modelo, como el experiencial-humanista, que se centra en el tratamiento idiosincrásico, que pone énfasis en la especificidad de cada ser humano y de cada proceso terapéutico (que excluye los manuales estandarizados que tal metodología reclama, con operaciones-recetas al estilo médico) y la relativización del diagnóstico clásico (como el DSM), el reto se convierte en imposible, si no quiere traicionar sus principios de deontología profesional de operar en bien del protagonista de la terapia, y su convicción de que aquel diagnóstico no hace 
equiparables a las personas. Como señalan Feixas y Pucurull, citado por Cestari, 2016, "se toma la categoría diagnóstica como elemento organizador clave, como si todos los sujetos que 'comparten trastorno' fueran homogéneos, únicamente por ese motivo (Feixas y Pucurull, 2012. Ciertamente, si se comparan grupos de 30 personas como mínimo, hay más probabilidades de sacar conclusiones, pero solo eso, probabilidades, no evidencias (con personas de la misma cultura). Los graves problemas, tanto metodológicos-señalados a propósito de la eficacia-como éticos, dificultan las posibilidades de aplicar con honestidad el paradigma PBE a la investigación en Psicología Humanista.

De todas formas, esta metodología de investigación, aplicado a la psicoterapia, ya ha sido criticada por autores como Feixas y Pucurull que, muy acertadamente, señalan:

Aunque el paradigma mencionado (PBE) tiene sus múltiples utilidades, una de la críticas más importantes que podemos encontrar es que por su construcción o control artificial de variables a la hora de obtener la información, los resultados obtenidos en las investigaciones realizadas en contexto clínico, se alejan de las condiciones auténticas con las que los terapeutas se encuentran a diario, porque este tipo de estudios al azar, no reproducen las verdaderas condiciones que existen en un contexto psicoterapéutico. (Feixas y Pucurull, 2012) (Ibidem, p. 11).

Es por ello por lo que surge un nuevo paradigma de investigación: la Evidencia Basada en la Práctica (EBP):

El paradigma de la EBP se basa en un uso cuidadoso, prudente y explícito de la evidencia obtenida en el encuadre de la práctica, tomando decisiones acerca de la atención a cada paciente como individuo. Esto significa integrar diferentes aspectos como son la pericia clínica del terapeuta, la alianza terapéutica y las medidas de resultados obtenidas en la práctica misma (Margison et al., 2000; Barkham y Margison, 2007). (Ibidem,p. 15).

Este paradigma es más partidario de prescindir de la "eficacia universal" (en la que no cree) y centrarse en la efectividad de determinadas intervenciones:

La efectividad, elemento del paradigma de la EBP, se refiere a aquellas investigaciones cuyo diseño otorga un gran poder de generalización en sus resultados, ya que los mismos son obtenidos en la práctica clínica in situ (Feixas y Pucurull, 2012). Estos autores plantean que el enfoque de la EBP se encamina a mostrar cuales son los procedimientos que funcionan en la mejora de la calidad de la atención en situaciones prácticas de la vida real.

Este paradigma se sustenta en la evidencia que no es posible obtener con otros modelos de investigación, ya que centra la adquisición de datos en lo que sucede en la clínica real, y a partir de esta saca sus conclusiones (o sus posibles hipótesis). (Ibidem, p. 15).

El problema de este paradigma de investigación es, lógicamente, que para poder llegar a una generalización, se requieren una ingente cantidad de investigaciones (cualitativas, de caso único) en la misma dirección y coordinadas entre sí, lo 
que requiere una infraestructura propia de la salud pública, en la que la constatación del psicoterapeuta particular de la efectividad de determinados procedimientos, se pierde. En el caso de las psicoterapias humanistas, dada la poca receptividad de las instituciones públicas respecto a la misma, la demostración se vuelve imposible en la práctica, aunque el terapeuta, por decencia profesional, las aplique (puesto que está convencido de una efectividad que ha constatado en multitud de casos), ya que, como señala Cencillo:

"mientras la Física puede detenerse ante los límites de la medibilidad [...] las ciencias humanas, sobre todo en sus aplicaciones prácticas, terápicas, pedagógicas y sociopolíticas, no admiten espera y han de arbitrar otros recursos, con otras reglas de juego tal vez, para alcanzar sus fines de ayudar al hombre eficazmente, pues su eficacia no puede depender de una ortodoxia relativamente convencional, ni del cumplimiento reglamentario de unas vigencias de época propias de otras ciencias $\mathrm{y}$, en todo momento cuestionables" (Cencillo, 1977, p. 15).

Es por ello que los autores propugnan combinar los paradigmas PBE y EBP para poder garantizar un mayor conocimiento de las intervenciones terapéuticas más adecuadas. Insistiendo en que no se trata de modelos, de los que hasta el momento no tenemos constancia de la superioridad de uno sobre otro, salvo el estudio de Lizeretti (2009) acerca de la superioridad del modelo de Psicoterapia Integradora Humanista (Gimeno-Bayón y Rosal, 2016, 2017) sobre el cognitivoconductual para el tratamiento de la ansiedad.

\section{El Surgimiento de un Nuevo Paradigma}

Los psicólogos humanistas tienen presente que hace ya unos decenios se viene reclamando un nuevo paradigma epistémico para poder investigar esos procesos más complejos.

El gran físico Erwin Schrödinger, Premio Nobel por su descubrimiento de la ecuación fundamental de la mecánica cuántica (base de la física moderna), considera que la ciencia actual nos ha conducido por un callejón sin salida y que "la actitud científica ha de ser reconstruida, que la ciencia ha de rehacerse de nuevo" (1967). [...] Ilya Prigogine, por su parte, afirma que "estamos llegando al final de la ciencia convencional"; es decir, de la ciencia determinista, lineal y homogénea, y presenciamos el surgimiento de una conciencia de la discontinuidad, de la no linealidad, de la diferencia y de la necesidad del diálogo" (1994, pág. 40). Desde la década de los años cincuenta en adelante, se han replanteado en forma crítica las bases epistemológicas de los métodos y de la misma ciencia (Martínez Miguélez, 2004, n.1.).

Ya en el año 1955, el ilustre astrofísico Julius Robert Oppenheimer, sucesor de Einstein en su puesto en el Institute For Advanced Studies, habiendo sido invitado a participar en el encuentro anual de la APA (American Psychological Association), la más importante asociación científica internacional de la Psicología, advirtió al colectivo de psicólogos que "el peor de todos los posibles errores sería 
que la psicología fuera inducida a modelarse a sí misma sobre una física que ya no existe, que ha quedado completamente superada en el tiempo" (1956, p. 134, cit. en Martínez Miguélez, 2003, n. 4).

Los mismos físicos-filósofos que crearon la física moderna se debatieron en las primeras décadas de este siglo con este problema, al constatar que no podían conceptualizar la realidad del átomo sin estudiar a fondo la acción del observador sobre el objeto percibido (Ibidem, n. 9).

Conscientes de las limitaciones de la metodología científica convencional aunque reconociendo su validez para algunos objetos de investigación- los científicos de la Psicología Experiencial-Humanista iniciaron, ya en los años ochenta del siglo pasado, propuestas para un nuevo paradigma epistemológico. Se puede destacar, entre ellas, la obra colectiva de Reason y Rowan (Eds.) (1981) Human Inquiry. A Sourcebook of New Paradigm Research, con aportaciones de J. Heron, G. W. Allport, R. Harré, W. R. Torbert, S. Reinharz y M. Maruyama, entre otros. En cuanto a las aportaciones de Rice y Greenberg y colaboradores (Rice y Greenberg, 1984) del nuevo paradigma de investigación, han podido ya superar en algunos sectores académicos la fase de acogida, en especial respecto a interés prioritario por la investigación del proceso terapéutico y de los "episodios de cambio" y de centrar la atención de la actividad mental o mecanismos psicológicos específicos experimentados por el paciente en los "acontecimientos de cambio intrasesión".

Los autores de este escrito se acogen a los requisitos de este nuevo paradigma de investigación, que resultan más aceptables y adecuados para el marco de la Psicología Humanista y que se pueden resumir en los siguientes puntos:

1. Se trata de una investigación cooperativa, en la línea de lo reclamado entre otros por Heron (1981a): "En la forma completa de este enfoque, no sólo será el sujeto plenamente un coinvestigador sino que el investigador será también un cosujeto, que participa plenamente en la acción y experiencia que ha de investigarse" (Heron, 1981a, p.195).

2. La capacidad de alcanzar una elevada calidad de conciencia, por parte del psicoterapeuta y del investigador es un requisito para la validez de una investigación según proponen, p.e., Reason (1981), Heron (1981b), y Maslow (1966). Reason y Rowan establecen ocho principios para garantizar la validez en la investigación del nuevo paradigma, relacionándose los dos primeros con esta cuestión. Los formulan con estos términos:

Una investigación válida se apoya sobre todo en una conciencia de alta calidad por parte de los coinvestigadores (Reason y Rowan, 1981, p.245).

Dicha conciencia de alta calidad sólo puede mantenerse si los coinvestigadores se implican en algún método sistemático de desarrollo personal e interpersonal (Ibidem, p.246).

Sin haber vivido la experiencia de profundizar en el propio autoconocimiento y sin algún tipo de trabajo personal sistemático que haya facilitado entrar en 
contacto con procesos internos inconscientes, estos autores consideran difícil alcanzar la capacidad de atención y de conciencia requerida para una investigación válida sobre los procesos psicológicos de otros sujetos.

Maslow se refiere también al peculiar estado de la mente, que requiere el terapeuta y el investigador, para comprender lo que ocurre en la mente de estos sujetos. Solamente este peculiar modo de atención puede ser fiel al enfoque holista -en contraposición a reduccionista- requerido por el nuevo paradigma. Con su lenguaje más bien informal y exagerado, lo resume en estos términos:

Todo clínico sabe que en el proceso de conocer a otra persona es mejor mantener su cerebro fuera del camino, y escuchar de forma total, encontrarse totalmente absorbido, receptivo, pasivo, paciente, y expectante más bien que apremiante, rápido e impaciente. No ayuda estar midiendo, preguntándose, calculando o probando nuestras teorías categorizando o clasificando. Si su cerebro está demasiado ocupado, usted no puede escuchar y ver bien. El término de Freud atención flotante describe bien esta clase de conocimiento de otra persona que no interfiere, global, receptivo, que espera (Maslow, 1966, citado en Rowan, 1981, p.83).

3. El conocimiento experiencial es requerido, en la ciencia del nuevo paradigma, como complemento del conocimiento proposicional y del conocimiento práctico. Tanto Heron como Reason lo reclaman, como también Harré, Rowan, Torbert y Reinharz, entre los autores que colaboran en la obra ya citada de Reason y Rowan (1981):

Conocimiento experiencial es conocer una entidad -persona, lugar, pensamiento, proceso, etc.- en un encuentro e interacción cara a cara. Es conocer a una persona o cosa a través de un trato prolongado. La investigación empírica, precisamente porque es empírica, requiere necesariamente cierto grado de conocimiento experiencial sobre las personas y objetos sobre los que trata la investigación. Las conclusiones del investigador son proposiciones sobre personas o cosas de los cuales él o ella ha tenido un conocimiento experiencial a través de un encuentro directo (Heron, 1981b, p.27).

De los principios de Reason y Rowan, ya citados, para la validez de la investigación, el quinto contiene también esta reclamación: "La investigación válida implica una interacción sutil entre diferentes formas de conocimiento" (Reason y Rowan, 1981, p.249), en la que se refiere al logro de un conocimiento más "denso" y más "sustancial" cuando incluye las cuatro formas de conocimiento experiencial, práctico, presentacional y proposicional. Torbert subraya también este requisito con particular énfasis:

De acuerdo con el nuevo modelo de investigación, un sistema de actuación que no se ocupe de un estudio de sí mismo experiencial no puede ni producir ni reunir datos válidos debido a las incongruencias no examinadas en el interior de su experiencia. Tal sistema distorsionará los datos tanto de forma deliberada como sin querer y resistirá procesar un feedback que identifique las incongruencias (Torbert, 
1981, p.150).

El requisito del conocimiento experiencial, e incluso la preferencia del mismo, en este nuevo paradigma, no margina el tipo de conocimiento en el que se ha centrado la ciencia occidental: "Mi tesis es que este conocimiento experiencial es prioritario respecto al conocimiento verbal-conceptual pero que están integrados jerárquicamente y necesitan uno del otro" (Maslow, 1966, citado por Rowan, 1981, p.87).

Estos tres elementos que se han destacado aquí, entre los que constituyen requisitos de la investigación en el nuevo paradigma, han formado parte de la manera habitual de abordar el trabajo terapéutico e investigador de toda sesión clínica por parte de los autores y su entorno. En ella se considera importante ejercitar un trabajo como coinvestigadores y cosujetos, atender con la máxima receptividad posible con actitud holista, y disponer de una vivencia experiencial previa de los trabajos propuestos a la vez que se facilita, por parte de los investigadores, cierto grado de actitud experiencial en el transcurso de la sesión (Rosal y Gimeno-Bayón, 20013, pp. 26-28).

\section{Vicios y Trampas en la "Evidencia Científica"}

Más allá de la sacralización de la "evidencia científica" como la máxima cota de objetividad y veracidad, habrá que hablar también los vicios y trampas que se dan en ella y en torno a ella, que, en definitiva, se convierten en equivalente a fake news en publicaciones académicas o científicas que se suponen serias y rigurosas. No se va a ahondar en el tema, que daría para otro artículo, pero sí a apuntar algunos aspectos que contribuyen a una visión más realista de las publicaciones que dan lugar a lo que luego se llamará "evidencia científica".

Algunos de estos vicios y trampas son de tipo grosero, fruto de presiones ambientales. Otros, más sutiles, fruto del deseo de conservar el prestigio científico.

Empezando por los de tipo grosero, hay que decir que destacan, entre las presiones ambientales:

a) La presión académica. Hay que comprender, ante todo, las dificultades con las que algunos investigadores del campo de la salud se encuentran en la práctica para poder realizar este trabajo con la calidad requerida a una denominación tan pomposa y venerada como "evidencia científica" cuando, como demuestra Ioannidis (2018) la mayoría de los resultados de investigación publicados son falsos. Sin duda, bastantes profesores de universidad se ven obligados a publicar con una determinada periodicidad para poder mantenerse en sus puestos. Por ello, algunos de los mismos publican investigaciones con demasiada rapidez, sin el sosiego necesario para hacerlas en forma rigurosa y concienzuda.

b) Otros investigadores del campo de la salud, que reciben financiación de una determinada entidad (un laboratorio farmacéutico, por ejemplo) necesitan obtener unos resultados que complazcan a dicha entidad, pues de la publicación de la "evidencia científica" de la eficacia, eficiencia y efectividad de un medicamento 
depende la generación de importantes ganancias económicas. Con estas presiones, no todos pueden, quieren o saben resistir a las presiones. $\mathrm{Y}$ así nos encontramos con variantes que distorsionan los resultados de la investigación. Aquí se señalan solo algunos:

\section{El sesgo de publicación científica}

Fue a mediados del siglo pasado cuando Sterling definió el sesgo de publicación, indicando que los estudios con resultados estadísticamente significativos tienen más probabilidad de ser publicados. Mientras que estudios con resultados no significativos se publican en menor medida. Sterling dejaba patente los problemas que podían provocar este sesgo: investigadores que repiten estudios con resultados negativos, posibles errores en los estudios con resultados positivos que no serán discutidos por otros estudios de resultados negativos, etc.

Actualmente existen varios factores que inciden en el sesgo de publicación: (a) La decisión de los autores de no publicar los resultados de su estudio al no ser estos estadísticamente significativos. (b) El rechazo de los editores de las revistas a publicar un estudio con resultados negativos, aun teniendo la calidad metodológica ${ }^{2}$ suficiente para dar fiabilidad a los resultados. (c) La exclusión de este tipo de estudios en las búsquedas bibliográficas de otros investigadores (Rosa Garrido, 2016, p. 1)

El problema de este sesgo es que, el investigador apremiado por publicar, que realiza un estudio en el que no se confirma la hipótesis planteada, como tiene menos posibilidades de que se publique su trabajo -por más que es tan interesante confirmar como descartar una hipótesis, para la comprensión de la realidad-pasará a producir el "efecto cajón" archivando dicho estudio con los trastos viejos y volviéndolo a replicar hasta que se confirme la hipótesis publicando solo este trabajo, sin mencionar los otros, o bien emprendiendo la investigación de otras hipótesis hasta que la confirmación de alguna de ellas le de la ocasión de cumplir con las exigencias académicas o publicitarias.

El truco de los "informes registrados. En otros casos, la presión académica por publicar puede llevar al autor a publicar "informes registrados", en los que el investigador, en lugar de publicar un estudio ya realidad, presenta para su publicación el planteamiento y diseño del mismo, y justifica su relevancia. Si la revista lo admite y publica, ya no importa el resultado. El autor ha publicado un artículo y le cuenta en su curriculum. El resultado ¿a quién le importa? A él no, desde luego, como bien han advertido Nosek y Lakens (2014).

El sesgo de la investigación financiada. Muy pegado a este sesgo está el de la investigación financiada por una entidad con intereses mercantiles, que obliga al investigador a obtener los resultados que conducirán al consumo de determinados productos farmacéuticos, por ejemplo. Ello explica el hallazgo de Ioannidis, que había investigado durante unos cuantos años las verdades y falsedades en el campo de las publicaciones científicas, sobre todo en relación con la genética molecular, 
del desproporcionado número de confirmación de hipótesis cuando las investigaciones son financiadas.

El sesgo de confirmación, muy cerca del anterior, pero que no trata de complacer a quien financia la investigación, sino a a las propias creencias, escuela, etc. del investigador, generando los "falsos positivos" que denunciaban Simmons, Nelson y Simonshon (2011), y que llevan a Ioannidis (director del Centro de Innovación en Meta-Investigación de la Universidad de Stanford) a concluir:

Corolario 5: Cuanto mayores son los intereses y prejuicios financieros y de

otro tipo en un campo científico menor es la probabilidad de que los resultados

de la investigación sean verdaderos. Los conflictos de interés y los prejuicios pueden aumentar el sesgo $u$. Los conflictos de interés son muy comunes en la investigación biomédica (Krimsky, Rothenberg, Stott y Kyle, 1998), y suelen ser reportados en forma inadecuada y poco frecuente (ibid.; Papanikolaou, Baltogianni, Contopoulos-I. et al., 2001). Los prejuicios no necesariamente tienen orígenes financieros.

Los científicos de un campo dado pueden tener prejuicios, debido simplemente a su creencia en una teoría científica o al compromiso con sus propios resultados. Muchos estudios en apariencia independientes, realizados en las universidades, se pueden llevar a cabo únicamente para dar a los médicos e investigadores calificaciones para su promoción o su titularidad. Tales conflictos no financieros también pueden llevar a reportar resultados e interpretaciones distorsionados. Los investigadores prestigiosos pueden suprimir la aparición y la difusión de resultados que refuten sus hallazgos mediante el proceso de revisión por pares. Asi perpetúan falsos resultados en su campo de investigación. La evidencia empírica sobre la opinion de los expertos muestra, además, que es poco confiable (Antman, Lau et al., 1992) (Ioannidis, 2018, p. 303).

Camerer et al. (2018), a la hora de detectar falsos positivos señalan que, a la hora de replicar investigaciones, las creencias de replicabilidad de los compañeros están fuertemente relacionadas con la replicabilidad, lo que sugiere que la comunidad de investigación podría predecir qué resultados se replicarían y que los fallos en la replicación no fueron solo resultado del azar (Camerer et al, 2018)

El mercadeo profesor/alumno es otra de las trampas que pueden darse de forma no excepcional. Aquí no se trata de un fraude directo, como el antes nombrado de Stapel, sino indirecto: el profesor da como tarea a los alumnos, para aprobarles, que realicen una serie de entrevistas y pasaciones de cuestionarios y le entreguen el material para hacer su investigación. El alumno aprueba, el profesor tiene un material obtenido fácilmente, y todos contentos. Salvo que a veces, el alumno comodón o rebelde se inventa los resultados. Como además, el estudiante sabe qué clase de resultados le gustaría obtener al profesor, hace que los que ofrece confirmen lo que el profesor espera. $\mathrm{Y}$ en ese fraude se basan algunas investigaciones 
publicadas, que se dan por buenas.

Aparte de estos vicios y trampas groseros, hay otros más sutiles, entre los que solo se citarán dos:

EI truco de la redacción en tercera persona. Salvo el caso de la investigación cualitativa, en el que se utiliza la redacción en primera persona por parte del investigador, dejando claro que es una persona la que está detrás del artículo, en las revistas científicas, o se redacta el artículo en tercera persona, o no se publica. Eso significa que, sutilmente, se induce al lector a que piense que la investigación cuantitativa o el artículo, son más coincidentes con la realidad, al estar libres de la subjetividad de un autor, que ha desaparecido en el texto. Ya no se trata, ni siquiera, del "nos" mayestático a que nos tenía acostumbrado el antiguo lenguaje papal, pues ni siquiera se puede utilizar la primera persona del plural. El "se" se convierte así en una afirmación que pareciera darse por compartida por un número indeterminado de sujetos, como medio de imponer respeto e intimidar sutilmente al intelecto del lector.

La compraventa de citas es otro truco oculto para ganar prestigio científico. Como parte del mismo se obtiene de las veces que un autor es citado en las publicaciones de otros, el mercado de intercambio de citas florece en determinados círculos: yo te cito, o te hago citar, cinco veces en revistas de impacto, y tú me citas o me haces citar, cinco veces en ese mismo tipo de revistas. Fácil, ¿no?

La trampa de llamar "pseudociencia" a la que no sea la propia. Aunque esta sea la trampa que ha impelido a escribir este artículo, no se trata de nada nuevo. Se puede traer aquí un hermoso ejemplo del siglo XII:

"El susodicho anciano -Anselmo de Laón-roído por la envidia y azuzado por las instituciones contra mí mencionadas, empezó a perseguirme en mis lecciones de Escritura no menos que lo hiciera antes mi maestro Guillermo en las de filosofía. En las clases de este anciano había entonces dos estudiantes que parecían destacar sobre los demás. Eran Alberico de Rheims y Lotulfo de Lombardía, cuya hostilidad hacia mí era tanto más intensa cuanto más presumían de sí mismos. Su insinuación - como después se comprobó- hizo que el anciano, visiblemente trastornado, me impidiera continuar glosando la obra comenzada que yo desarrollaba en su cátedra, alegado que no quería que se le imputara a él ningún error que yo pudiera formular por mi falta de competencia. Cuando esto llegó a oídos de los estudiantes, su indignación no tuvo límites. Evidentemente, era una calumnia fruto de la rabia y de la envidia, cosa que nunca había sucedido antes con ningún otro." (Cartas de Abelardo y Eloísa, Historia calamitatum, cap II, p. 45).

La denigración del que tiene seguidores por aquellos que querrían tener exclusiva de estos, es tan antigua -seguramente- como la historia humana. Bien a principio de ella sitúa la Biblia el fratricidio de Caín. Los científicos no están libres de la tendencia cainita y si, además, hay intereses económicos o de prestigio académico de por medio, es fácil que esta trampa tome la forma de degradar la categoría intelectual o científica de los otros. Y parece que tanto el Ministerio de 
Salud como el de Ciencia han caído en la trampa urdida por organizaciones médicas y laboratorios farmacéuticos, a quienes les vendría muy bien que desaparecieran los psicólogos humanistas. Los propios colegas de otras corrientes se han sumado a esta denigración y han formado coro con los funcionarios ministeriales, y las organizaciones médicas para calificar a la Psicología Humanista de pseudociencia. ¿O se trata solo de ignorancia? Quién sabe.

\section{Conclusiones}

Todas estas reflexiones sobre lo que significa "evidencia científica" en el campo de la Psicología y la psicoterapia, así como la puesta de relieve de sus dificultades y miserias, forman parte de los aspectos a tener en cuenta a la hora de tildar a la Psicología Humanista, y los modelos en ella encuadrados, de pseudociencia. Eso es lo que pretenden algunas listas acientíficas que están manejando el Ministerio de Sanidad, Consumo y Bienestar Social, y el Ministerio de Ciencia, Innovación y Universidades, en la elaboración de su Plan para la Protección de la Salud frente a las Pseudoterapias. La expresión "evidencia científica" citada con desparpajo nada menos que veinticinco veces en un texto de once folios, se merece, como mínimo, una explicación acerca de lo que los ministerios autores del plan entienden por ella, en el caso de las psicoterapias. Se merece, también, que justifique en qué evidencia científica se basa la confección de las listas que maneja, cuando la más "seria" de ellas, procedente de un concienzudo estudio oficial de 2011 (en la que incluye a algunas psicoterapias humanistas como "terapias naturales" susceptibles de ser pseudociencias), reconoce en su nota 1 al anexo en que figuran, que la lista se ha elaborado "a partir de la información extraída de diversas páginas de Internet". $\mathrm{Ni}$ hace falta entrar en las otras listas, pensando en que esta es la más rigurosamente documentada.

Pues bien: si nos vamos a otras listas, como la que tiene la American Psychologial Association, que es la institución más prestigiosa en el ámbito internacional en Psicología y psicoterapia, se encontrarán que en la División 32 se encuentra albergada (desde 1970) la Psicología Humanista. Y si los ministerios consultan la lista de presidentes de dicha asociación, se encontrará con algunos psicólogos humanistas, como Maslow, Rogers o Bugental. Y si consultan la lista que recoge dicha asociación en su Resolución de 9 de agosto de 2012, como grandes categorías dentro de la psicoterapia, se encontrarán que allí figuran (junto con la terapia conductual, la cognitiva, el psicoanálisis y terapias dinámicas, y la terapia integrativa u holista) las terapias humanistas. Y si además leen la resolución, señala que en el texto "se define la posición de la APA en lo que respecta a la consideración de la psicoterapia basada en la evidencia" concluyendo que "de acuerdo con los estudios científicos, estas intervenciones psicológicas deben ser reconocidas por el sistema sanitario como prácticas eficaces y rentables en relación con sus costes/ beneficios". Tal reconocimiento lo hace para las cinco categorías, sin excepción, teniendo en cuenta más de 140 estudios de calidad y metaanálisis "en ensayos 
clínicos controlados y en contextos reales, así como los resultados obtenidos en diversas poblaciones".

En relación con el Focusing, un metaanálisis de 89 estudios (Hendricks, 2001) mostró cómo la adquisición de las habilidades propias del mismo está relacionada con el éxito en la terapia, con independencia de la cultura y orientación terapéutica en que se inserten.

En otro metaanálisis realizado por Elliot, respecto a 86 estudios, se muestra cómo las psicoterapias humanistas son eficaces a largo plazo "En los ensayos clínicos aleatorios con pacientes de control de tratamiento comparativo, los clientes en terapias humanísticas generalmente muestran cantidades de cambio equivalentes a los clientes en terapias no humanistas, incluida la terapia cognitiva conductual" (Elliot, 2002).

Finalmente, se puede citar, por ejemplo, el metaanálisis realizado por Elliot y Freire en 2008 respecto a las terapias rogerianas (TCC), en el que se analizaron 191 estudios, que en conjunto sumaban más de 14.000 personas, y en los que -por seleccionar una de las comparaciones realizadas-la Terapia Centrada en la Persona/ Experiencial daba los mismos resultados en cuanto a eficacia que la Terapia Cognitivo Conductual.

Todos estos metaanálisis hacen que se pueda afirmar, con Elliot, Greenberg y Lietaer: "La evidencia ahora es lo suficientemente sólida como para que podamos recomendar que las terapias experiencialhumanísticas se consideren tratamientos con apoyo empírico." (Elliot, Greenberg y Lietaer, 2004).

Y ahora viene la pregunta: ¿Va a tener más peso científico, para los ministerios implicados en el Plan antes citado, la opinión del funcionario encargado de realizar una búsqueda por internet que todas las investigaciones y metaanálisis citados? ¿Van a tener más peso las presiones de instituciones con conflictos de intereses en relación con la psicoterapia (que no receta medicación alguna, aunque pueda colaborar con médicos y psiquiatras que sí la recetan), o con un modelo determinado de psicoterapia que pretende eliminar del mapa de las ciencias a las psicoterapias humanistas, entre otras, para tener menos competencia?

¿Van, por ello, a seguir situando determinadas instituciones profesionales a las psicoterapias humanistas entre las técnicas que no van a incidir en la salud mental, sino que "van fundamentalmente dirigidas al bienestar o confort del usuario" (documento de análisis de situación de las terapias naturales del Ministerio de Sanidad, Política social e Igualdad de 2011 al que se ha hecho referencia)? ¿O a incluirlas entre las pseudoterapias, como hace indirectamente el Consejo General de la Psicología de España (CGCOP) cuando parece que hace un "acto de tolerancia" hacia ellas al decir que "algunas de las que pueden ser consideradas pseudoterapias pueden suponer beneficios contrastados para la salud de los pacientes, cuando son utilizadas correctamente por profesionales psicólogos en el marco de una adecuada relación terapeuta-paciente?" (CGCOP, Infocop, 02/01/2019)

Todo ello no parece muy científico, pero...el futuro dirá si prevalece la ciencia, 
o las diferentes instituciones políticas, corporativas, mercantiles e institucionales (incluida las instituciones académicas) prefieren, presionan, o consienten que se impongan otros factores.

\section{Referencias Bibliográficas}

Also, A. (2017, marzo). Programa piloto de psicoterapia en trastornos de ansiedad para de prescribir benzodiazepinas. (Trabajo de doctorado en la Facultad de Medicina de la Universidad de Barcelona). Comunicación presentada en las IV Jornadas de la Asociación de Psicoterapia Integradora Humanista, Barcelona, España.

Anguera, M. T. (1981a). La observación (I): Problemas metodológicos. En R. Fernández Ballesteros y J. A. I. Carrobles (Eds.), Evolución conductual: Metodología y aplicaciones (pp. 292-333). Madrid, España: Pirámide.

Anguera, M. T. (1981b). La observación (II): Situaciones naturales y de laboratorio. En R. Fernández Ballesteros y J. A. I. Carrobles (Eds.), Evaluación conductual: Metodología y aplicaciones (pp.334-363). Madrid, España: Pirámide.

Anguera, M. T. (1986). La investigación cualitativa. Educar, 10, 23-50.

Anguera, M. T. (1995). Metodología cualitativa. En M. T. Anguera, J. Arnau, M. Ato, R. Martínez, J. Pascual y G. Vallejo (Eds.), Métodos de investigación en psicología (pp. 515-576). Madrid, España: Síntesis

American Psychological Association (APA) (2012). Las intervenciones psicológicas son eficaces y rentables. En Infocop Online. Recuperado de: http://www.infocop.es/view article.asp? id $=4184$

Barkham, M. y Margison, F. (2007). Practice-based Evidence as a Complement to Evidence-based Practice: From Dichotomy to Chiasmus. En C. Freeman y M. Power (Eds.) Handbook of Evidence-based Psychotherapies: A Guide for Research and Practice (pp. 443-476). West Sussex, Reino Unido: John Wiley.

Berne, E. (2010). La intuición yel Análisis Transaccional. Sevilla, España: Jeder. (Traducción del original en inglés de 1977).

Camerer, C. F., Dreber, A., Holzmeister, F., Ho, T-H., Huber, J., Johannesson, M.,... Wu, J. (2018) Evaluating the replicability of social science experiments.in Nature and Science between 2010 and 2015. Nature Human Behaviour, 2, 637-644. Recuperado de: https:/www.nature.com/articles/s41562-018-0399-z

Caparrós, A. (1978). La psicología, ciencia multiparadigmática, Anuario de Psicología, 19, 80-109.

Caparrós, A. (1980). Los paradigmas en psicología (Sus alternativas y sus crisis). Barcelona, España: Horsori.

Cartas de Abelardo y Eloísa (1993). Trad. De P. R. Santidrián y M. Astruga. Madrid, España: Alianza Editorial.

Cencillo, L. (1977). Transferencia y sistema de psicoterapia. Madrid, España: Pirámide.

Cencillo, L. (1978). El hombre. Noción cientifica. Madrid, España: Pirámide.

Cestari, P. (2016). Paradigmas de investigación en psicoterapia (Trabajo fin de grado, Universidad de la República de Paraguay, Paraguay). Recuperado de: https://bit.ly/2UB74TY

Charman, D. y Barkham, M. (2005). Psychological treatments: Evidence-based practice and practice-based evidence. Recuperado de: http://psychology.org.au/publications/inpsych/treatments/

Consejo General de la Psicología España (2019, enero). El Consejo General de la Psicología hace aportaciones al Plan de Protección de la Salud frente a las Pseudoterapias. Infocop. Recuperado de: http://www.infocop.es/ view article.asp?id=7866

Corey, G. (1995). Teoría y Práctica de la Terapia Grupal. Bilbao, España: Desclée de Brouwer.

Elliot, R. y Freire, E. (2014) Las terapias centradas en la persona/experienciales son altamente efectivas: Resumen del Metaanálisis de 2008. En A. S. Segrera, H. D. Jeffrey, Cornelius-White, Behr, M. y S. Lombardi (Eds.) Consultorías y psicoterapias centradas en la persona y experienciales (pp. 105-112). Buenos Aires, Argentina: Gral Aldea Editores.

Elliot, R., Greenberg, L. S. y Lietaer, G. (2004). Research on Experiential Psychotherapies. En M. J. Lambert (Ed.) Bergin and Garfield's Handbook of Psychotherapy and Behavior Change (5th ed.) (pp. 493-539). Oxford, Reino Unido: John Wiley.

Feixas, G. y Pucurull, O. (2012). Psicoterapeutas en formación en atención primaria: un estudio naturalístico. Segundo premio FEAP 2012. Recuperado de: http://feap.es/index.php/federacion/premios-feap

Gimeno-Bayón, A. (2013). Un modelo de integración corporal de la psicoterapia. Lérida, España: Milenio. 
Gimeno-Bayón, A. y Rosal, R. (2 ${ }^{\mathrm{a}}$ ed.) (2016). Psicoterapia Integradora Humanista. Manual para el tratamiento de 33 problemas psicosensoriales, cognitivos y emocionales. Bilbao, España: DDB.

Gimeno-Bayón, A. y Rosal, R. (2017). Manual Práctico de Psicoterapia Integradora Humanista. Tratamiento de 69 problemas en los procesos de valoración, decisión y práxicos. Bilbao, España: DDB.

Hendricks, M. (2001). Focusing-Oriented/Experiential Psychotherapy. En D. Cain, D. y J. Seeman (Eds.) Humanistic Psychotherapy: Handbook of Research and Practice (pp. 221-251). Washington, DC.: American Psychological Association.

Heron, J. (1981a). Experiential research methodology. En P. Reason y J. Rowan (Eds.), Human inquiry. A source book of new paradigm research (pp. 153-166). Nueva York, NY: John Wiley.

Heron, J. (1981b). Philosophical basis for a new paradigm. En P. Reason y J. Rowan (Eds.), Human inquiry. A source book of new paradigm research (pp. 19-36). Nueva York, NY: John Wiley.

Hoffman, L. (1987). Fundamentos de la terapia familiar. Ciudad de México, México: Fondo de Cultura Económica.

Ioannidis, J. P. A. (2018). Por qué la mayoría de los resultados de investigación publicados son falsos. Revista de Economía Institucional, 20(39), 297-313. https://doi.org/10.18601/01245996.v20n39.13

James, W. (1989). Principios de Psicología. Ciudad de México, México: Fondo de Cultura Económica.

Krimsky, S., Rothenberg, L. S., Stott, P. y Kyle, G. (1998). Scientific journal and their authors' financial interests: A pilot study. Psychotherapy and Psychosomatic, 67(4-5), 194-201.

Lam Díaz, R. M. y Hernández Ramirez, P. (2008). Los términos: eficiencia, eficacia y efectividad ¿son sinónimos en el área de la salud? Rev Cubana de Hematología, Inmunología y Hemoterapia 24(2). Recuperado de: http://scielo.sld.cu/scielo.php?script=sci_arttext\&pid=S0864-02892008000200009

Lambert, M. J. y Bergin, A. E. (1994). The effectiveness of psychotherapy. En A. E. Bergin y S. L. Garfield (Eds.), Handbook of psychotherapy and behavior change (4a ed.) (pp. 143-189). Nueva York, NY: Wiley.

Lambert, M. J. Shapiro, D. A. y Bergin, A. E. (1986). The effectiveness of psychotherapy. En S. L. Gardfield y A. E. Bergin (Eds.), Handbook of psychotherapy and behavior change ( $3^{\mathrm{a}}$ ed.). Nueva York, NY: Wiley.

Lizeretti, N. P (2009). Tratamiento de los Trastornos de Ansiedad: Diseño y Evaluación de una Intervención Grupal basada en la Inteligencia Emocional (Tesis doctoral, Universidad Ramon Llull, Barcelona, España). Recuperado de: https://www.tesisenred.net/handle/10803/9269

Luborsky, L., Singer, B. y Luborsky, L. (1975). Comparative studies of psychotherapies. Archives of General Psychiatry, 32, 995-1008.

Makel, M. C., Plucker, J. A. y Hegarty, B. (2012). Replications in psychology research:how often do they really occur? Perspectives on Psychological Science 7(6), 537-542.

Margison, F. R., Barkham, M., Evans, C., McGrat, G., Mellor, C., Audin, K. y Connell, K. Measurement and psychotherapy: Evidence-based practice and practice-based evidence. The British Journal of Psychiatry, $177(2), 123-130$.

Martínez Miguélez, M. (2003). Sobre el estatuto epistemológico de la Psicología. Polis, Revista Latinoamericana 4. Recuperado de: https://journals.openedition.org/polis/7188

Martínez Miguélez, M. (2004). El proceso de nuestro conocer postula un nuevo paradigma epistémico. Polis, Revista Latinoamericana, 8. Recuperado de: https://journals.openedition.org/polis/6170

Maslow, A. (1966). The psychology of science. A reconnaissance. Nueva York, NY: Harper and Row.

Ministerio de Sanidad, Política Social e Igualdad (2011). Análisis de situación de las terapias naturales. Recuperado de: http://www.mscbs.gob.es/novedades/docs/analisisSituacionTNatu.pdf

Mullings, B. (2017). A literature review of the evidence for the effectiveness of experiential

psychotherapies. Melbourne, Australia: PACFA.

Nosek, B. A. y Lakens, D. (2014). Registered reports. A method to increase the credibility of published result. Social Psychology, 45, 137-141. https://doi.org/10.1027/1864-5335/a000192

Oppenheimer, R. (1956). Analogy in science. American Psychologist, 11, 127-135.

Oriol, M.F. (2014, abril). ¿Es la PIH constructora de esperanza?: una aproximación empírica. Comunicación presentada en las III Jornadas de la Asociación de Psicoterapia Integradora Humanista, Barcelona, España.

Papanikolaou, G. N., Baltogianni, M. S., Contopolos Ioannidis, D. G., Haidich, A-B., Giannakakis, I. A. y Ioannidis, J. PA. (2001). Reporting of conflicts of interest in guidelines of preventive and therapeutic interventions. BMC Mediacal Researh Methodology, 1, 3.

Paul, G. L. (1967). Strategy of outcome research in psychotherapy. Journal of Consulting Psychology, 31, 109-118.

Reason, P. y Rowan, J. (1981). Human Inquiry. A Sourcebook of New Paradigm Research. Nueva York, NY: John Wiley \& Sons. 
Rice, L. y Greenberg, L. S. (1984). Patterns of change: Intensive analysis of psychotherapeutic process. Nueva York, NY: Guilford.

Rosa Garrido, M. C. (2016). Editorial. Sesgo de publicación: ¿existe también en estudios de metanálisis? Index de Enfermería, 25(1-2). Recuperado de: http://scielo.isciii.es/scielo.php?script=sci_arttext\&pid=S113212962016000100002

Rosal, R. (1997). La efectividad de la actividad imaginaria para el cambio terapéutico creativo. Bilbao, España: Universidad de Deusto.

Rosal, R. (2001). Por qué y para qué surgió el Movimiento de la Psicología Humanista. En A. Gimeno-Bayón y R. Rosal, Cuestiones de Psicología y Psicoterapias Humanistas (pp. 17-40). Barcelona, España: Instituto Erich Fromm de Psicoterapia Integradora Humanista. $2^{\mathrm{a}}$ reimpresión en 2013, Lérida, España: Milenio.

Rosal, R. (2012). El poder psicoterapéutico de la actividad imaginaria y su fundamentación científica. Barcelona, España: Instituto Erich Fromm de Psicoterapia Integradora Humanista.

Rosal, R. (2013). Las actitudes del terapeuta. En A. Gimeno-Bayón y R. Rosal, Cuestiones de Psicología y Psicoterapias Humanistas (pp. 191-207). Lérida, España: Milenio.

Rosal, R. y Gimeno-Bayón, A. (2013). Memoria Académica del Instituto Erich fromm de Psicoterapia Integradora Humanista (actualizada en marzo de 2013). Barcelona, España: Instituto Erich Fromm de Psicoterapia Integradora Humanista.

Rowan, J. (1981). The psychology of science, by Abraham Maslow. En P. Reason y J. Rowan (Eds.), Human Inquiry. A sourcebook of New Paradigm research (pp.87-92). Nueva York, NY: John Wiley.

Shea, C. (2011). Fraud Scandal Fuels Debate Over Practices of Social Psychology. The Chronicle of the Higher Education, 58(13). Recuperado de https://www.chronicle.com/article/As-Dutch-Research-Scandal/129746/

Simmons, J. P., Nelson, L. D. y Simonshon, U. (2011). False-Positive Psychology: Undisclosed Flexibility in Data Collection and Analysis Allows Presenting Anything as Significant. Psychological Science. https://doi.org/ 10.1177/0956797611417632

Torbert, W. R. (1981). Why educational research has been so uneducational: the case for a new model of social science based on collaborative inquiriy. En P. Reason y J. Rowan (Eds.), Human inquiry. A sourcebook of New Paradigm research (pp. 141-152). Nueva York, NY: John Wiley.

Varios: Open Science Collaboration (2015) Science, 349(6251). https://doi.org/10.1126/science.aac4716

Vera-Villarroel, P. y Mustaca, A. (2006). Investigaciones en Psicología Clínica Basadas en la Evidencia en Chile y Argentina. Revista Latinoamericana de Psicología, 38(3), 551-565. Recuperado de: https:// www.redalyc.org/articulo.oa?id=80538308 